\title{
Assessment of the nutritional status of severely ill COVID-19 patients and possible risk factors
}

In December 2019 COVID-19, a respiratory illness caused by the severe acute respiratory syndrome coronavirus 2 (SARS- CoV-2), appeared in Wuhan, China. Nutritional status is considered an indicator of "resilience against destabilization" (1). Inadequate nutritional intake contributes to adverse health outcome amongst COVID-19 patients ${ }^{(2)}$. Nutrition assessment and treatment effectively reduce complications and improve clinical outcomes ${ }^{(3)}$ for hospitalized severely ill patients. The current study aimed to assess the nutritional status of COVID-19 severely ill patients in Al Madinah Al Munawarah, Saudi Arabia.

An epidemiological, cross-sectional study included severely ill COVID-19 patients admitted to the General and Ohud Hospitals. Information about demographic characteristics included questions to assess their gender, age, education level and marital status. Nutrition screening was conducted using the Malnutrition Screening Tool (MST). Weight and height were measured for the calculation of body mass index (BMI). Patients were asked about the presence of symptoms such as nausea, vomiting, diarrhoea, constipation, fever, loss of appetite, weight loss, and decreased food intake. Data collectors wore Personal Protective Equipment before working with patients and spent a maximum of 15 minutes with each patient. Ethical approval was obtained from Taibah University and the Ministry of Health. All patients consented before the start of the study. Descriptive statistics, Pearson's correlation, and Multiple Regression were determined using SPSS version 26. Significance level was set at $<5 \%$.

A total of 123 patients (30\% females) were included. Most patients aged 51-65-year-old (40\%), were married ( $83 \%$ ), and were Saudi $(16 \%)$. They had diabetes and hypertension (16\%) and suffered from nausea, vomiting, fever, cough and diarrhoea (53\%). Patients complained from loss of appetite $(77 \%)$, weight loss $(42 \%)$, and decreased food intake $(50 \%)$. Patients $(52 \%)$ had low malnutrition risk, $43 \%$ at risk and 5\% had high risk. Overweight was common among $36 \%$ and $24 \%$ were obese. The risk of malnutrition correlated positively with loss of appetite $(\mathrm{r}=0.49, \mathrm{P}<0.0005)$ and decreased food intake $(\mathrm{r}=0.38, \mathrm{P}<0.0005)$ and weight loss $(\mathrm{r}=0.95, \mathrm{P}=$ 0.0005). Multiple Regression revealed that appetite loss $($ Beta $=0.171, \mathrm{CI}=0.127-0.368)$, weight change $(0.932$, CI $=0.657-0.769)$ and BMI $(0.149, \mathrm{CI}=0.014-0.206)$ affected

patients' nutritional status (determined by MST).

Overall, a significant number of patients were at risk or high risk of malnutrition. Possible factors included loss of appetite, which is affected by decreased food intake, leading to a change in weight. The BMI affected the nutritional status of patients putting them at increased risk of malnutrition. Nutrition screening is an essential step which helps to determine patients who could benefit from detailed nutrition assessment. Early detection of malnutrition reduces hospital stay and cost of medication. It speeds up the rate of recovery and improves health outcomes.

\section{Acknowledgments}

Thank you to all the nurses who helped with data collection. Thank you to the patients and to Abdelmoez Moawia Labib for data entry

\section{References}

1. Naja F and Hamadeh R (2020) Eur J Clin Nutr, 74, 1117-1121.

2. Wu C., Chen X., Cai Y., et al. (2020) JAMA Intern Med. 180(7):934-943.

3. Barazzoni R, Bischoff SC, Breda J et al., (2020) Clin Nutr 39;1631-8. 